\title{
NOUVELL
}

\section{Distribution géographique de la drépanocytose en 2010}

Frédéric B. Piel
University of $0 x f o r d$,

Tinbergen building,

Department of Zoology,

South Parks Road, OXI 3PS,

Oxford, Royaume-Uni.

fred.piel@zoo.ox.ac.uk

www.eeid.ox.ac.uk
> La drépanocytose est une maladie génétique de l'hémoglobine. Cette maladie est causée par la mutation d'un seul nucléotide et a été décrite pour la première fois dans la littérature scientifique il y a plus d'un siècle [1]. Malgré tout, les données épidémiologiques concernant sa distribution géographique actuelle et le nombre d'individus atteints à l'échelle globale sont limitées. L'objectif de cet article est de décrire les avancées méthodologiques récentes qui permettent de combler partiellement ces lacunes.

Le mécanisme de sélection du gène responsable de la drépanocytose est bien connu. Un individu homozygote normal, porteur d'hémoglobine $A$ (génotype $A A$ ), est sain mais susceptible de mourir du paludisme dans les régions endémiques. Un individu hétérozygote pour la mutation (génotype AS) ne souffre d'aucun symptôme associé à la drépanocytose mais bénéficie d'une protection élevée contre le paludisme. Un individu homozygote pour la mutation (génotype SS) est atteint de la drépanocytose. Dans ce cas, les propriétés physiques des globules rouges sont altérées et une réduction de leur élasticité conduit à leur stagnation dans les vaisseaux sanguins, ce qui induit généralement des crises vaso-occlusives, des dommages à différents organes (notamment la rate, le cœur, les poumons et les reins) et une susceptibilité accrue aux infections bactériennes [1]. Il n'y a pas, à l'heure actuelle, de traitement définitif pour la drépanocytose et cette maladie est souvent létale dans les pays en voie de développement. Un diagnostic précoce, par exemple grâce à un programme de dépistage néonatal, permet de prévenir le développement de la plupart des complications médicales associées à cette maladie. L'éducation et le conseil génétique sont d'autres outils importants de prévention et de contrôle de la drépanocytose. Cependant, afin d'adopter des mesures de santé publique adaptées, il est nécessaire de connaitre la distribution géographique et le nombre de personnes affectées par cette maladie.

\section{Distribution géographique de la drépanocytose}

L'hypothèse selon laquelle des fréquences élevées du gène drépanocytaire étaient observées dans les régions endémiques pour le paludisme a été formulée à la fin des années 1940 [2] et nous l'avons récemment confirmée par une étude géographique détaillée (Figure 1A) [3, 4]. Ce gène était originellement prévalent dans les populations exposées au paludisme en Afrique sub-saharienne, dans le bassin méditerranéen (notamment en Grèce et en Italie), au Moyen-Orient et dans certaines parties du subcontinent indien. Suite à d'importants mouvements de populations, délibérés ou forcés, au cours des siècles derniers, la distribution du gène drépanocytaire s'est progressivement étendue indépendamment de celle du paludisme, principalement le long des côtes orientales des Amériques, dans les Caraïbes et en Europe de l'Ouest. Grâce à une étude rigoureuse de la littérature scientifique, nous avons pu assembler une base de données épidémiologiques sur la drépanocytose, comprenant 1211 études de populations locales. L'utilisation des géostatistiques a permis ensuite d'estimer la fréquence allélique du gène drépanocytaire dans les régions pour lesquelles les données épidémiologiques sont rares ou absentes, et ainsi de générer des cartes géographiques (Figure 1B). Les fréquences alléliques les plus élevées, de l'ordre de $18 \%$, ont été observées uniquement au nord de l'Angola. Des fréquences supérieures à $10 \%$ ont été estimées en Afrique centrale et en Afrique de l'Ouest, ainsi que dans certaines parties de l'Arabie Saoudite et de l'Inde.

\section{Estimation des populations touchées par la drépanocytose}

Un programme de dépistage universel permet d'obtenir des données précises sur le nombre d'individus affectés par une maladie génétique. Les États-Unis et le Royaume-Uni ont mis en place de tels programmes pour les nouveau-nés [5]. Dans les autres pays, les données de prévalence sont limitées aux informations fournies par les études locales des populations, souvent conduites dans les années 1960 ou 1970. Extrapoler la prévalence de la drépanocytose pour un pays à partir de la moyenne d'un nombre limité d'études fournit des estimations très approximatives. Les méthodes de cartographie et de statistiques modernes permettent de développer des modèles mathématiques probabilistes prenant en compte la taille des échantillons des populations testées, leur distribution géographique et les densités de populations locales. Cette méthodologie permet de générer des estimations contemporaines 


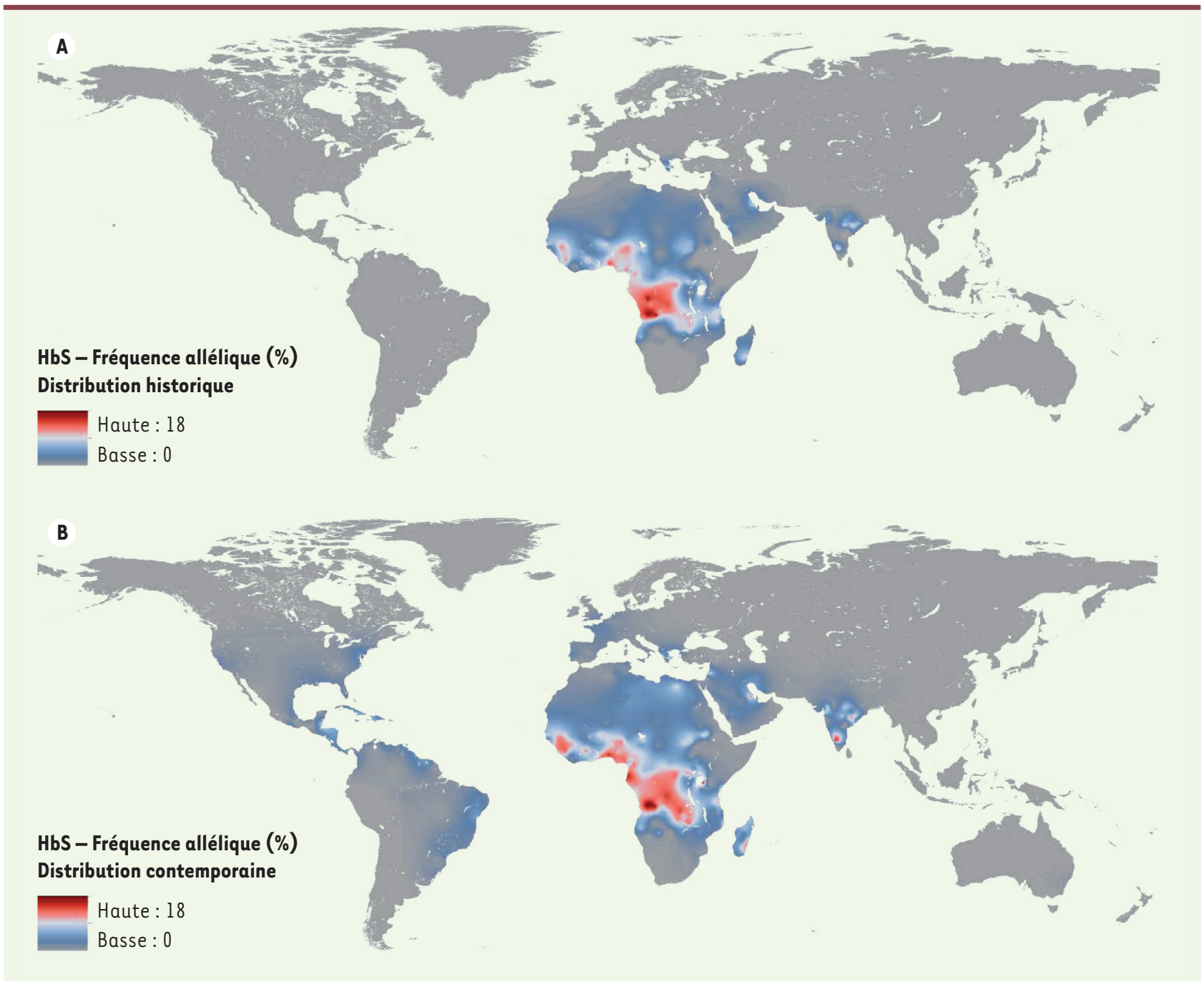

Figure 1. Distribution historique et contemporaine (2010) de la drépanocytose. A. Les données de distribution historique sont extraites de [3]. B. Les données de distribution contemporaine (2010) sont extraites de [5]. HBS : hémoglobine S pour sickle-cell disease (dénomination anglaise de la maladie).

avec des échelles nationale, régionale et mondiale, accompagnées d'une mesure de leur précision (intervalle de confiance ou intervalle interquartile ${ }^{1}$ ). Sur la base des données de prévalence locale publiées dans la littérature scientifique, nous avons donc pu estimer le nombre de naissances d'individus porteurs du gène drépanocytaire et de malades atteints de la drépanocytose pour chaque pays en 2010. Au total, nous avons estimé le nombre de nais-

${ }^{1}$ L'intervalle interquartile correspond à l'étendue de la série statistique après l'élimination de $25 \%$ des valeurs les plus faibles et de $25 \%$ des valeurs les plus fortes. sances d'individus AS et SS, respectivement, à 5476000 (intervalle interquartile : $5291000-5679000$ ) et 312000 (294 000 - 330000$)$ [6]. La vaste majorité des nouveau-nés atteints se situent en Afrique ( $75 \%$ des SS), et seulement 1 $\%$ en Europe. Dans un contexte de globalisation et de mobilité croissante des populations à l'échelle mondiale, il est cependant de plus en plus fréquent pour les praticiens en Europe d'être confrontés à des cas de porteurs du gène drépanocytaire, voire à des malades drépanocytaires. Il est dès lors essentiel de pouvoir informer ces patients des risques pour leurs descendants, et de leur offrir des conseils génétiques et un soutien psychosocial adéquats.

\section{Conclusion}

Il est important de continuer à effectuer des études épidémiologiques afin de déterminer les besoins actuels et futurs en terme de politique de santé publique propre à la drépanocytose, tant à l'échelle mondiale qu'à l'échelle nationale. Aucun pays d'Afrique n'a, à l'heure actuelle, mis en place un programme systématique de dépistage national de la drépanocytose, et les données existantes pour certaines régions du Nigeria, 
de la République Démocratique du Congo ou de l'Inde sont encore très limitées. Dans les pays ayant implémenté de tels programmes, il est essentiel de mesurer régulièrement les progrès effectués afin, à la fois, de démontrer leur efficacité et de continuer à les améliorer. La drépanocytose représente un problème de santé publique à l'échelle mondiale, qui reste négligé jusqu'à présent [7]. $\diamond$ Sickle-cell disease: geographical distribution and population estimates

\section{LIENS D'INTÉRÊT}

L'auteur déclare n'avoir aucun lien d'intérêt concernant les données publiées dans cet article.

\section{RéFÉRENCES}

1. Rees DC, Williams TN, Gladwin MT. Sickle-cell disease. Lancet 2010 ; 376 : 2018-31.

2. Haldane JBS. The rate of mutation of human genes. Hereditas $1949 ; 35: 267-73$.

3. Piel FB, Patil AP, Howes RE, et al. Global distribution of the sickle cell gene and geographical confirmation of the malaria hypothesis. Nat Commun 2010 ; 1 : 104.

4. Labie D. Les relations complexes entre hémoglobinopathies et paludisme. Med Sci (Paris) $2010 ; 26: 685-7$
5. Streetly A, Clarke M, Downing M, et al. Implementation of the newborn screening programme for sickle cell disease in England: results for 2003-2005. J Med Screen 2008 ; 15 : 9-13.

6. Piel FB, Patil AP, Howes RE, et al. Global epidemiology of sickle haemoglobin in neonates: a contemporary geostatistical model-based map and population estimates. Lancet 2013 ; 381 : 142-51.

7. Weatherall DJ. The inherited diseases of hemoglobin are an emerging global health burden. Blood 2010; $115: 4331-6$.

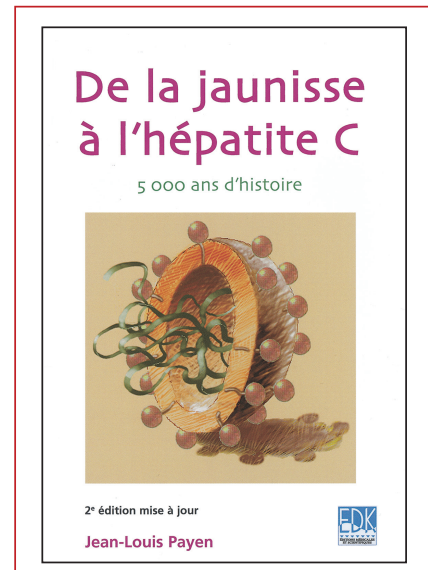

ISBN : 978-2-8425-4136-1 128 pages

a jaunisse est un symptôme facilement identifiable ; il paraissait bien naturel que l'homme, confronté à une modification de la couleur de ses yeux et de sa peau ait de tous temps recherché les causes de cette transformation.

II n'est donc pas surprenant que le premier traité de médecine, écrit 3000 ans avant J.C. par un médecin sumérien, décrive déjà la jaunisse. À chaque époque de l'histoire de la médecine, les praticiens, influencés par les concepts médicaux de leur temps, attribuèrent une ou plusieurs explications particulières à ce symptôme. Ainsi, du démon Ahhâzu des Sumériens à la sophistication des biotechnologies qui permirent la découverte du virus de l'hépatite $C$, le lecteur cheminera sur une période de 5000 ans au travers des différents continents.

Ici encore, l'histoire se révèle une formidable source de réflexion : le foie souvent impliqué dans l'apparition des jaunisses est-il le siège de l'âme?

Les expérimentations humaines chez des volontaires ou chez des enfants handicapés mentaux étaient-elles justifiées pour permettre la découverte des virus des hépatites?

Le formidable développement de la transfusion sanguine, des vaccinations, mais aussi de la toxicomanie explique-t-il les épidémies d'hépatites du XXe siècle?

Autant de questions qui sont abordées dans ce livre passionnant et accessible à tous.

U À retourner à EDK, 25, rue Daviel - 75013 Paris - Tél. : 0158101905 - Fax : 0143293262 - E-mail : edk@edk.fr

NOM :

Prénom :

Adresse :

Code postal :

Ville :

Pays :

Fonction :

Je souhaite recevoir l'ouvrage De la jaunisse à l'hépatite $\mathbf{C , ~} \mathbf{5} 000$ ans d'histoire : $12 €+3 €$ de port $=\mathbf{1 5} € \mathbf{T T C}$

en ................ exemplaire, soit un total de

$€$

U $\square$ Par chèque, à l'ordre de $\mathbf{E} \mathbf{D} \mathbf{K}$

○ Par carte bancaire : $\square$ Visa $\square$ Eurocard/Mastercard

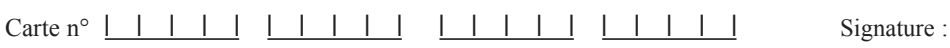

Date d'expiration: $\quad$ । $111 \quad$ । $1 \quad 1$

N $\mathrm{N}^{\circ}$ de contrôle au dos de la carte :

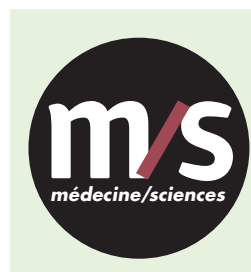

Tarifs d'abonnement $m / s-2013$

$>$ Grâce à $m / s$, vivez en direct les progrès

des sciences biologiques et médicales

Abonnez-vous

à médecine/sciences

Bulletin d'abonnement page 968 dans ce numéro de $\mathrm{m} / \mathrm{s}$

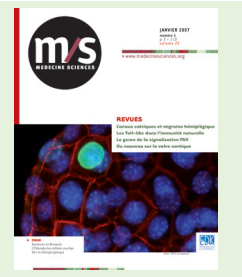

